MODELING, IDENTIFICATION AND CONTROL, 1983, VOL. 4, NO. 3, 175-194

doi:10.4173/mic.1983.3.4

\title{
Parameter estimation of ship manoeuvring equations
}

\author{
TORE FLOBAKK†
}

Keywords: parameter estimation, hydrodynamics, captive and free-running model testing.

Model tests performed at the Norwegian Hydrodynamic Laboratories (NHL), to identify ship manoeuvring equations, are described. Parameter estimation techniques are used to determine the mathematical model.

\section{Introduction}

Traditionally, model testing has been used to predict full scale manoeuvring properties of ships in two ways:

The scale model is controlled to perform the same manoeuvres which are to be predicted in full scale. Froude scaling of the results is then used to estimate the full scale manoeuvres.

The parameters in mathematical models, defined as a set of differential equations, are estimated by least squares fitting of results from planar motion or rotating arm testing. The mathematical model itself, is the required result in some applications, for example, for representation of a ship in a training simulator. In other situations, the mathematical model is used to simulate the manoeuvres to be predicted.

A planar motion mechanism and rotating arm facility are quite expensive, and economical arguments motivate a search for alternative methods for determination of mathematical models. Another argument is that the available methods are restricted to model testing.

The purpose of the work presented in this paper has been to use parameter estimation techniques to determine the mathematical model. The tests performed are free-running tests, and also oblique towing tests with a quite simple test setup.

At the time the tests were done, November 1980, the towing tank was the only available laboratory unit within NHL, where manoeuvring tests could take place. This tank is $250 \times 10 \mathrm{~m}$ and $6 \mathrm{~m}$ deep. Because of the limited width of the tank, zig-zag manoeuvres with small rudder deflections were the only freerunning model tests that could be performed. This test was a modified zig-zag manoeuvre, designed to let the model move around the centre line of the tank. Rudder deflections up to 10 degrees were used. This was a restriction, since it could not be expected that the non-linear effects would be significant. The system identification methods used to identify parameters were the least squares method and the maximum likelihood method. These methods are described in Åstrøm and Eykhoff (1971), Sælid (1975) and Hallingstad (1976). Similar methods have earlier been used to identify parameters from full scale trials: Astrøm, Källstrøm, Norrbin and Byrstøm (1975), Blanke (1978) and Källstrøm (1979).

Received 25 July 1983.

$\dagger$ Norwegian Hydrodynamic Laboratories, P.O. Box 4118, Valentinlyst, N-7002 Trondheim, Norway. 


\section{Mathematical models}

Depending on the application, various structures of mathematical models of manoeuvring ships have been proposed. The complexity of these models, represented by the number of parameters used, varies from 2 or 3 to 50 or 60 .

Some common applications of mathematical models are:

Autopilots

Dynamic positioning systems

Training simulators.

Models can be organized according to the areas of manoeuvring they cover:

Service speed in unrestricted waterways: This is a typical application for an autopilot, with the aim to minimize fuel consumption. Simple linear models are used.

Reduced speed in restricted waterways and near harbours: When automatic control systems are used, the aim is to increase safety. In this case fuel consumption becomes less important.

Low or zero speed: Situations like offshore loading and dynamic positioning systems. Usually relatively simple non-linear models are used.

External forces caused by wind, waves and current can be represented in all the above situations, and in addition, models for sailing in restricted waterways may include shallow water and wall effects.

Application of mathematical models in training simulators is demanding and requires the most complex models, covering all speed ranges and external forces. On the other hand, tuning of the parameters is usually done according to a class of ships rather than to a particular ship. The mathematical models used in this paper are restricted to those covering service speed in unrestricted waterways. External forces caused by wind, waves and current are not covered. This means that the forces to be discussed are:

Inertia forces,

Hydrodynamic forces on the hull,

Control forces from rudder and thrusters.

With coordinate system definitions as given in Fig. 1, the inertia forces for movements in 3 degrees of freedom (surge, sway and yaw) are well known from the literature (Abkowitz 1964, Norrbin 1970). The linearized model is of the form:

$$
\left(\begin{array}{cc}
m-Y_{\dot{v}} & m x_{g}-Y_{\dot{r}} \\
m x_{g}-N_{\dot{v}} & I_{0}-N_{\dot{r}}
\end{array}\right)\left(\begin{array}{c}
\dot{v} \\
\dot{r}
\end{array}\right)=\left(\begin{array}{cc}
Y_{v} & Y_{r}-m u_{0} \\
N_{v} & N_{r}-m x_{g} u_{0}
\end{array}\right)\left(\begin{array}{l}
v \\
r
\end{array}\right)+\left(\begin{array}{c}
Y_{\delta} \\
N_{\delta}
\end{array}\right) \delta
$$

where

$$
\begin{array}{ll}
m & \text { mass of ship } \\
I_{0} & \text { moment of inertia } \\
x_{g} & \text { position of the centre of gravity relative to the midship section } \\
u_{0} & \text { forward (surge) speed }
\end{array}
$$


$v$ sway speed

$r$ yaw speed

$\delta$ rudder angle

$Y$ lateral force

$N$ turning moment

$Y_{v}, Y_{r}$ etc. hydrodynamic derivatives $\left(Y_{\delta}=\frac{\partial Y}{\partial \delta}\right)$

The surge equation disappears in the linear model. For ships bow-stern symmetry justifies simplification of the mass matrix (on the left hand side of eqn. (1)), since the off-diagonal elements are much smaller than those on the diagonal.

These equations refer to the local coordinate system on the ship. Including the coordinate system transformation to the global (earthfixed) coordinate system the

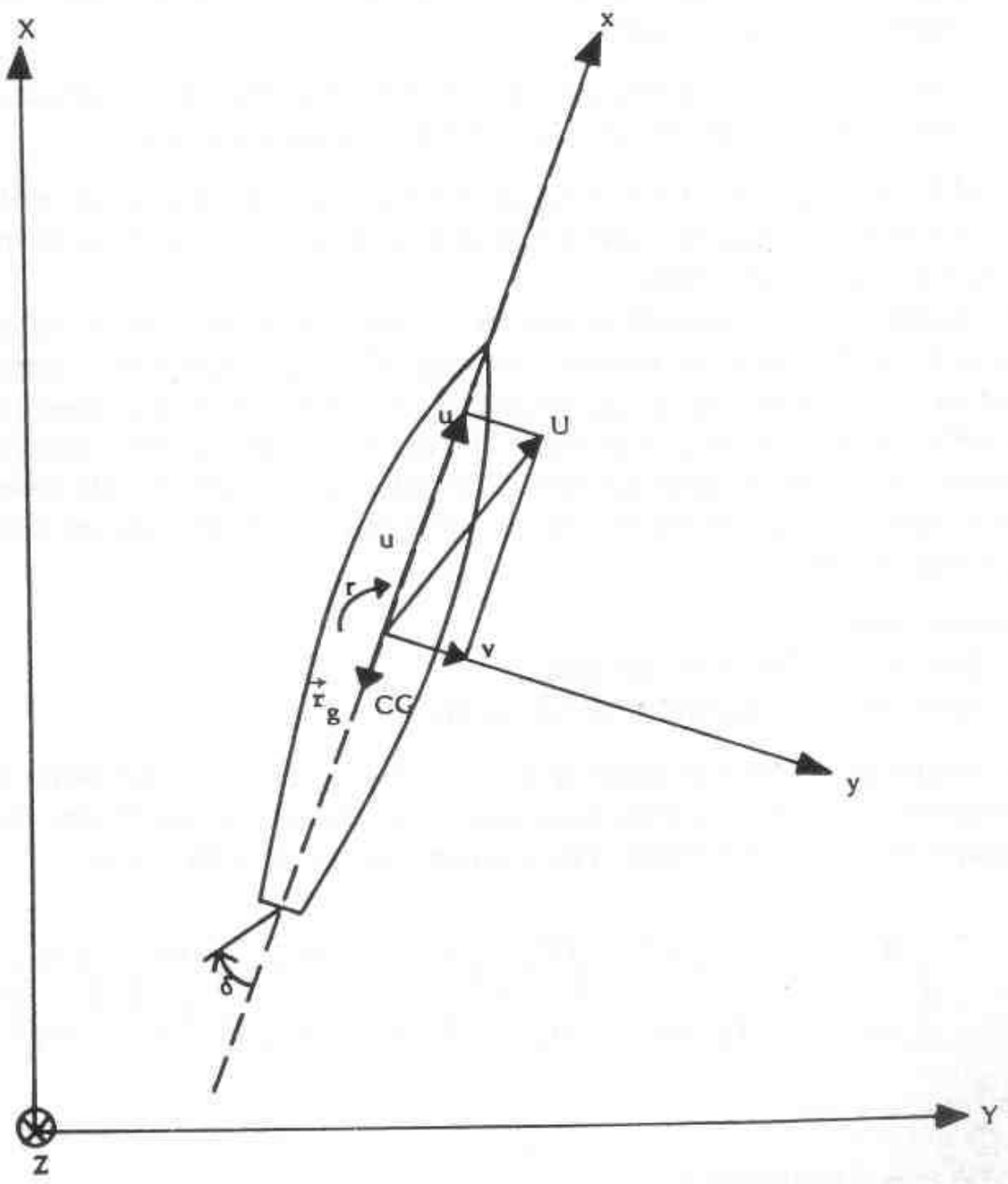

Figure 1. Coordinate system definitions. $X Y Z$ is an earth-fixed coordinate system and $x y z$ is a coordinate system with origin at the midship section following the ship. $U$ is total speed, $u$ surge speed, $v$ sway speed and $r$ yaw speed. $r_{g}$ is the vector in the $x y z$ system from the origin to the centre of gravity, $(C G)$. 
model takes the form

$$
\begin{aligned}
& \dot{u}=0 \\
& \dot{v}=\frac{1}{m-Y_{\dot{v}}}\left(Y_{v} v+Y_{r}+Y_{\delta} \delta\right) \\
& \dot{r}=\frac{1}{I_{0}-N_{\dot{r}}}\left(N_{v} v+N_{r} r+N_{\delta} \delta\right) \\
& \psi=r \\
& \dot{x}=u \cos \psi-v \sin \psi \\
& \dot{y}=u \sin \psi+v \cos \psi
\end{aligned}
$$

The terms $Y_{r}$ and $N_{r}$ here represent both hydrodynamic damping caused by yaw speed and also inertia effects $\left(m u_{0}\right.$ and $\left.m x_{\theta} u_{0}\right)$.

Further:

$\psi$ heading angle

$x, y$ position of ship in global coordinate system

With some modifications this model covers the model structures used in this paper.

\section{Model test}

The tests were performed with a model of a $127 \mathrm{~m}$ gas tanker in scale 1:21.377. Ship characteristics are given in Table 1 . All tests were done with a model speed $U=1 \cdot 112 \mathrm{~m} / \mathrm{s}$ corresponding to 10 knots in full scale. By means of a propulsion test

\begin{tabular}{|c|c|c|c|}
\hline & Symbol & Model & Ship \\
\hline Length between perpendicular & $L_{\mathrm{pp}}[\mathrm{m}]$ & $5 \cdot 941$ & $127 \cdot 00$ \\
\hline Length on water line & $L_{\mathrm{WL}}[\mathrm{m}]$ & $6 \cdot 192$ & $132 \cdot 36$ \\
\hline Breath moulded & $B \quad[\mathrm{~m}]$ & 0.959 & $20 \cdot 50$ \\
\hline Draught at $L_{\mathrm{pp}} / 2$ & $T \quad[\mathrm{~m}]$ & $0 \cdot 292$ & $6 \cdot 25$ \\
\hline Draught at FP & $T_{\mathrm{FP}}[\mathrm{m}]$ & $0 \cdot 187$ & $4 \cdot 00$ \\
\hline Draught at AP & $T_{\mathrm{AP}}[\mathrm{m}]$ & 0.398 & $8 \cdot 50$ \\
\hline Volume of displacement & $\nabla \quad\left[\mathrm{m}^{3}\right]$ & $1 \cdot 113$ & 10869 \\
\hline Block coefficient & $C_{\mathrm{B}}$ & 0.641 & 0.641 \\
\hline Wetted surface & $S \quad\left[\mathrm{~m}^{2}\right]$ & $6 \cdot 724$ & 3073 \\
\hline
\end{tabular}
the propeller revolutions $(n)$ at the self-propulsion point was found

$$
n=6 \cdot 67 \mathrm{rev} / \mathrm{s} \text {. }
$$

Two types of tests are described:

Captive model tests,

Free-running model tests.

Table 1. Principal hull data. Hull model no. M-1374; Model scale 1:21·377. 


\subsection{Captive model tests}

The model was instrumented with propeller and rudder, and fixed to a mechanism which could keep the model in various positions relative to the towing carriage. Transducers mounted on this mechanism were able to measure the forces transmitted to the model (Fig. 2).

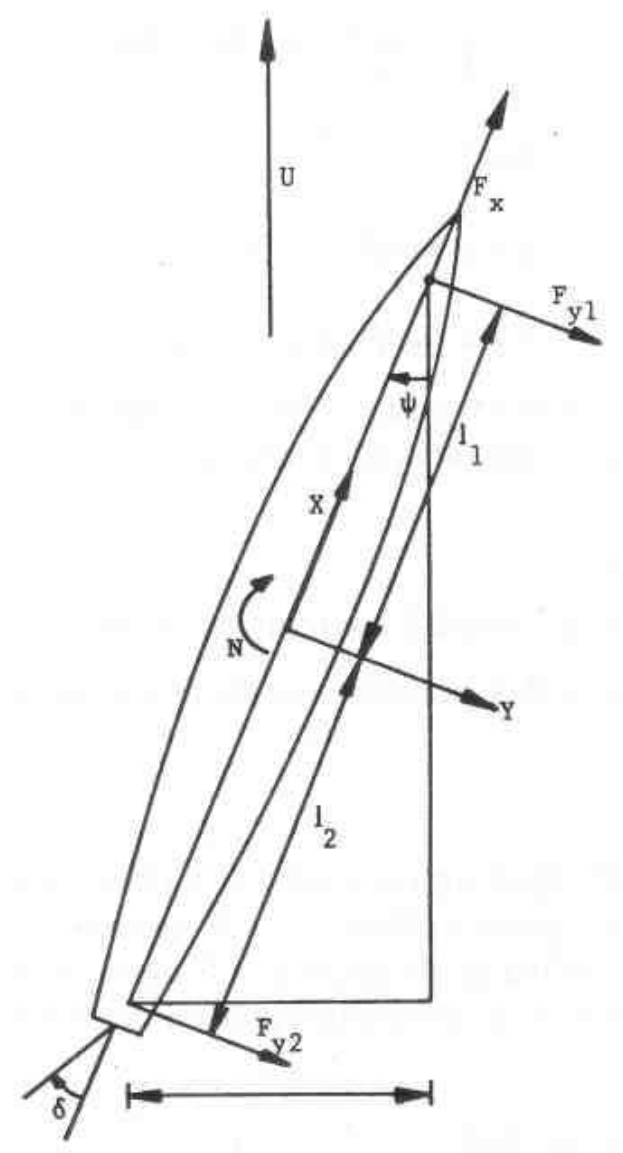

Figure 2. Measurement setup during captive model tests.

With this equipment, oblique towing tests in calm water were performed and the following quantities measured
Rudder angle
(ס) [degrees]
Propeller revolutions
(n) $[\mathrm{rev} / \mathrm{s}]$
Heading angle
( $\psi$ [degrees]
Total model speed
(Towing carriage speed)
(U) $[\mathrm{m} / \mathrm{s}]$
Lateral force at the bow
$\left(F_{y 1}\right)[N]$
Lateral force at the stern
$\left(F_{y 2}\right)[N]$ 
Knowing the distances $l_{1}$ and $l_{2}$ (Fig. 2), the quantities
Lateral force
(Y) $[N]$
Turning moment
(N) $[\mathrm{Nm}]$
Sway speed
(v) $[\mathrm{m} / \mathrm{s}]$

were calculated as follows:

$$
\left.\begin{array}{l}
Y=F_{y 1}+F_{y 2} \\
N=l_{1} F_{y 1}-l_{2} F_{y 2} \\
v=-U \sin \psi
\end{array}\right\}
$$

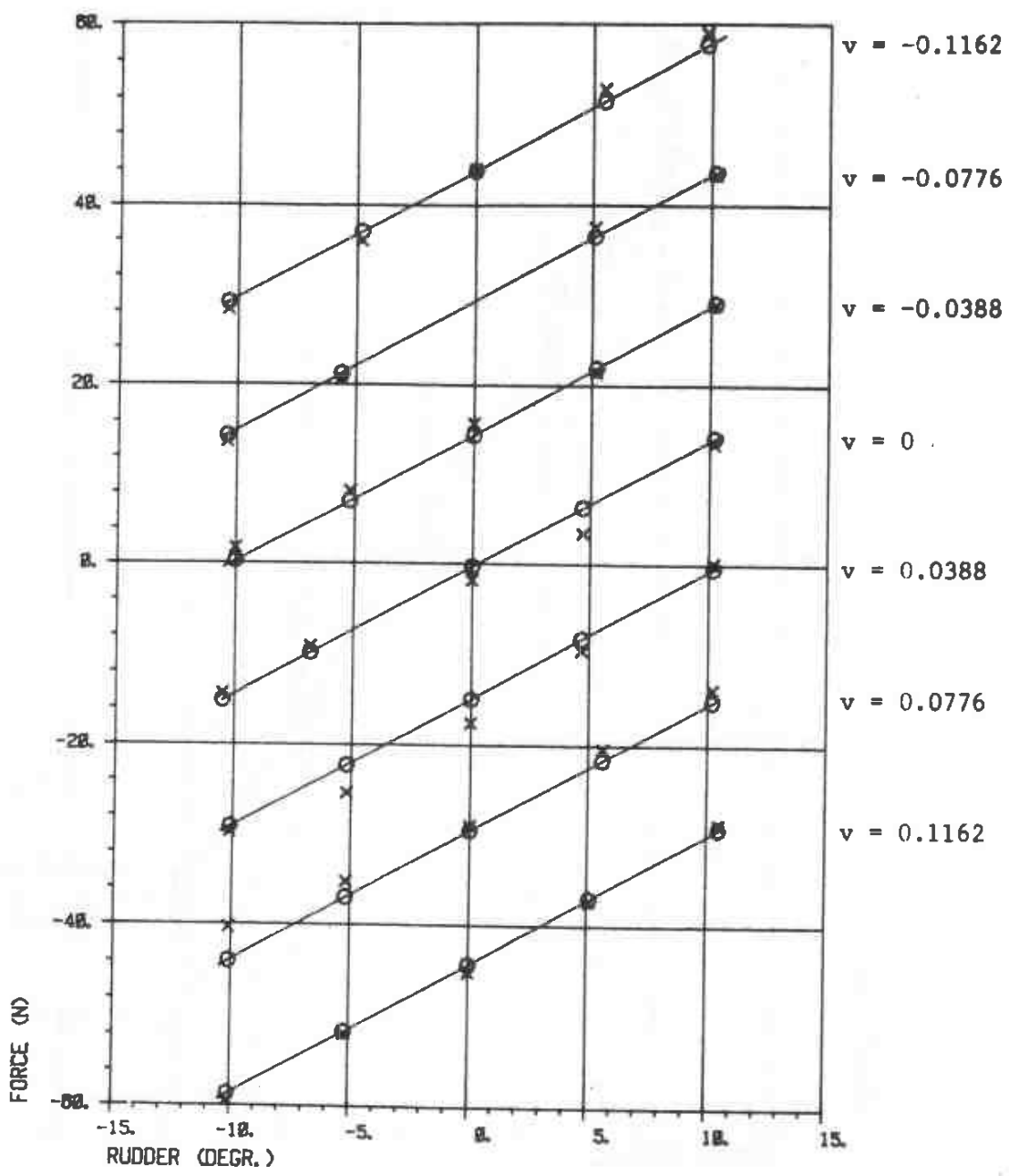

Figure 3. Captive model test results. The lateral force $Y(v, \delta)$ is plotted as a function of the rudder angle $\delta$ and sway speed $v, x$ indicates the measured values of $Y(v, \delta)$. These are fitted to the polynomial $Y(v, \delta)=Y_{v} \cdot v+Y_{\delta} \cdot \delta+Y_{0}$. The lines show $Y(v, \delta)$ as a function of $\delta$ for 7 values of sway speed, and $O$ indicates the fitted values corresponding to the measurements. 
The purpose of the oblique towing test was to determine the relations

$$
\left.\begin{array}{c}
Y=Y(v, \delta) \\
N=N(v, \delta)
\end{array}\right\}
$$

by perturbing heading angle and rudder angle. The test program included 34 such perturbations. A least squares algorithm was applied to estimate the influence of sway speed and rudder angle on lateral force and turning moment. The selected structure of the equations was:

$$
\left.\begin{array}{l}
Y(v, \delta)=Y_{v} v+Y_{\delta} \delta+Y_{0} \\
N(v, \delta)=N_{v} v+N_{v|v|} \cdot v|v|+N_{\delta} \delta+N_{0}
\end{array}\right\}
$$

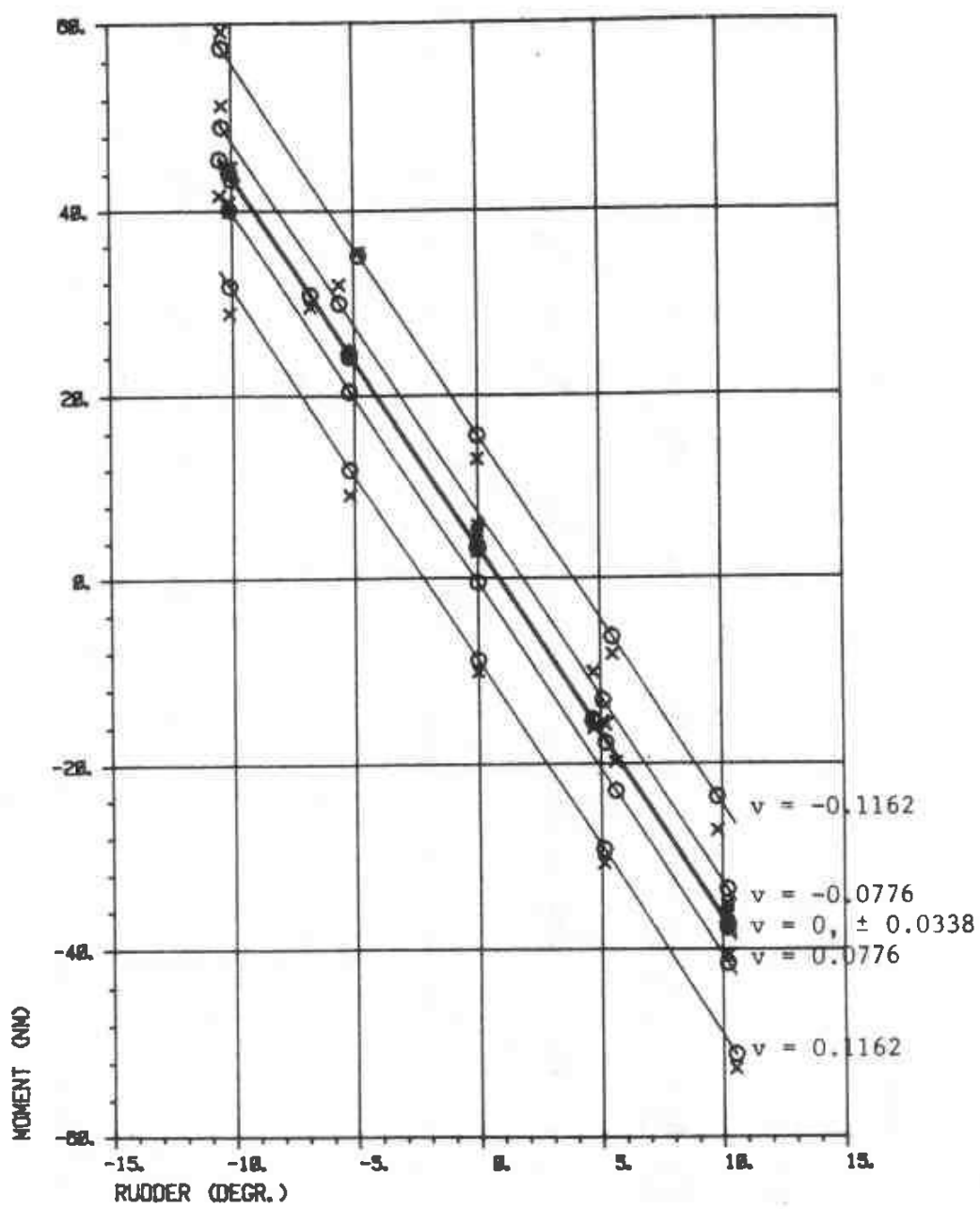

Figure 4. Captive model test results. The turning moment $N(v, \delta)$ is plotted as a function of the rudder angle $\delta$ and sway speed $v . \times$ indicates the measured values of $N(v, \delta)$. These are fitted to the polynomial $N(v, \delta)=N_{v} \cdot v+N_{v|v|} \cdot v|v|+N_{\delta} \cdot \delta+N_{0}$. The lines show $N(v, \delta)$ as a function of $\delta$ for 7 values of sway speed, and $O$ indicates the fitted values corresponding to the measurements. 
The bias terms $Y_{0}$ and $N_{0}$ are included to take up the non-symmetric effects from the propeller wake on the rudder.

Figures 3 and 4 give a graphic presentation of the measured values and fitted polynomials in eqn. (5). As observed from these figures, $Y$ is well represented by linear relationship in both $v$ and $\delta$. In the moment equation a non-linear term $N_{v|v|} \cdot v|v|$ is included to cover viscous cross-flow effects (Norrbin 1970).

\subsection{Free-running model tests}

During the free-running model tests, the model was free to move according to the forces acting on it, caused by propeller, rudder and hydrodynamic damping. The instrumentation is shown in Fig. 5. The measured quantities were
Rudder angle
( $\delta$ ) [degrees]
Yaw speed
(r) $[$ degrees $/ \mathrm{s}]$
Heading angle
$(\psi)$ [degrees]
Coordinates of model in global coordinate system $(x, y)[\mathrm{m}]$

To obtain the heading angle and the global coordinates an underwater acoustic measurement system was used (Rossing 1981). Transmitting hydrophones send an acoustic burst into the water, and receiving hydrophones detect these bursts. Timing electronics measure the time for the burst to reach the receivers. A configuration of 2 transmitting $\left(T_{1}\right.$ and $\left.T_{2}\right)$, and 2 receiving $\left(R_{1}\right.$ and $\left.R_{2}\right)$ hydrophones gave the distances $l_{1}, l_{2}, l_{3}$ and $l_{4}$ on Fig. 5 . With measurement of the position of the towing carriage $\left(x_{c}\right)$ the above quantities were calculated.

The tests were performed at the self-propulsion point of the model. The rudder was controlled according to a modified zig-zag manoeuvre algorithm to prevent the model from running into the tank walls. This consisted of letting the rudder turn from one side to the other according to a criterion being a linear combination of heading angle and distance from the centre line of the tank, instead of just according to the heading angle which is used during an ordinary zig-zag manoeuvre. Five runs numbered 221-225 were selected for analysis. Of these 5 runs, run number 221 was chosen for an initial analysis. The measurements for this run are presented on Fig. 6 .

The first approach was using run number 221 to identify the model

$$
\left.\begin{array}{l}
u_{k+1}=u_{k} \\
v_{k+1}=v_{k}+\tilde{Y}_{v} v_{k}+\tilde{Y}_{\delta} \delta_{k}+\tilde{Y}_{0} \\
r_{k+1}=r_{k}+\tilde{N}_{r} r_{k}+\tilde{N}_{\delta} \delta_{k}+\tilde{N}_{0} \\
\psi_{k+1}=\psi_{k}+\Delta t r_{k} \\
x_{k+1}=x_{k}+\Delta t\left(u_{k} \cos \psi_{k}-v_{k} \sin \psi_{k}\right) \\
y_{k+1}=y_{k}+\Delta t\left(u_{k} \sin \psi_{k}+v_{k} \cos \psi_{k}\right)
\end{array}\right\}
$$

without using the information from the captive model tests. Comparing eqn. (6) 
Parameter estimation of ship manoeuvring equations

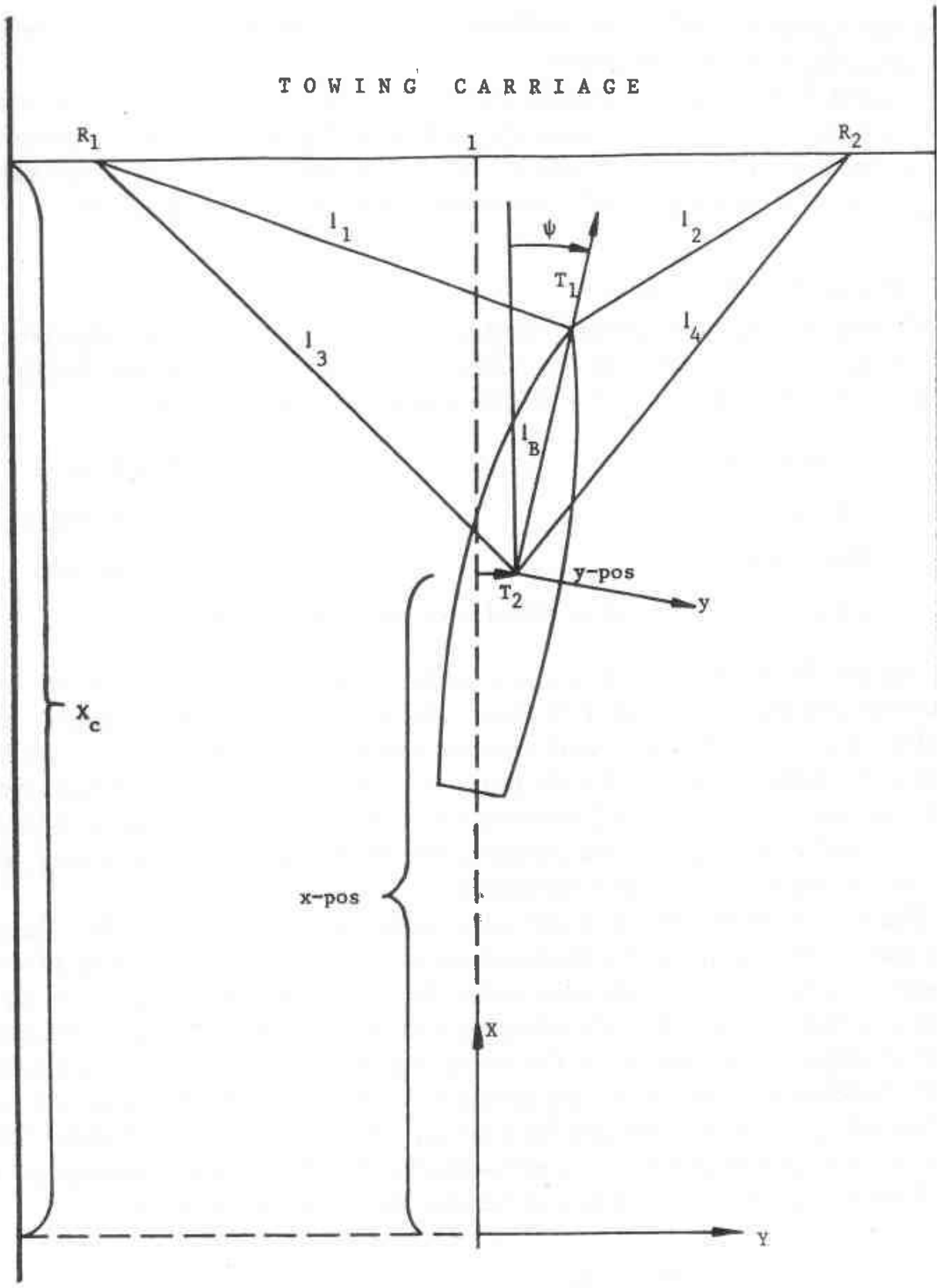

Figure 5. Measurement setup and coordinate definitions during free-running model 1

with eqn. (2), note that:

$$
\left.\begin{array}{l}
\tilde{Y}_{v}=\frac{Y_{v} \cdot \Delta t}{m-Y_{v}}, \quad \tilde{Y}_{\delta}=\frac{Y_{\delta} \cdot \Delta t}{m-Y_{v}} \\
\tilde{N}_{r}=\frac{N_{r} \cdot \Delta t}{I_{0}-N_{t}}, \quad \tilde{N}_{\delta}=\frac{N_{\delta} \cdot \Delta t}{I_{\delta}-N_{j}}
\end{array}\right\}
$$

where $\Delta t$ is the integration step length. 
The parameter vector

$$
\boldsymbol{\theta}=\left[\tilde{Y}_{v}, \tilde{Y}_{\delta}, \tilde{Y}_{0}, \tilde{N}_{r}, \tilde{N}_{\delta}, \tilde{N}_{0}\right]^{T}
$$

was identified using the maximum likelihood method. The parameter values estimated were used as input to the Tables 2 and 3.

In this simplified model $Y_{r}$ and $N_{v}$ are excluded from eqn. (2), and the parameters $\tilde{Y}_{v}$ and $\tilde{N}_{r}$ represent the time constants of the equations.

From these parameters it is obvious that the inertia parameters $m, Y_{\dot{v}}, I_{\delta}$ and $N_{\dot{\phi}}$ could not be determined.

For this reason the results from the captive tests were included as a priori information. The structure of the mathematical model used in this case was:

$$
\begin{aligned}
& u_{k+1}=u_{k} \\
& v_{k+1}=v_{k}+\frac{\Delta t}{m-Y_{v}}\left(Y_{v} v_{k}+Y_{r} r_{k}+Y_{\delta} \delta_{k}+Y_{0}\right) \\
& r_{k+1}=r_{k}+\frac{\Delta t}{I_{0}-N_{r}}\left(N_{v} \cdot v_{k}+N_{v|k|} \cdot v_{k}\left|v_{k}\right|+N_{r} \cdot r_{k}+N_{\delta} \cdot \delta_{k}+N_{0}\right) \\
& \psi_{k+1}=\psi_{k}+\Delta t \cdot r_{k} \\
& x_{k+1}=x_{k}+\Delta t\left(u_{k} \cos \psi_{k}-v_{k} \sin \psi_{k}\right) \\
& y_{k+1}=y_{k}+\Delta t\left(u_{k} \sin \psi_{k}+v_{k} \cos \psi_{k}\right)
\end{aligned}
$$

In this model the parameter vector known from the captive tests was:

$$
\boldsymbol{\theta}=\left[Y_{v}, Y_{\delta}, N_{v}, N_{v|v|}, N_{\delta}\right]^{T}
$$

The remaining parameters to be determined from the free-running tests were:

$$
\boldsymbol{\theta}=\left[\frac{\Delta t}{m-Y_{\dot{v}}}, Y_{r}, Y_{0}, \frac{\Delta t}{I_{0}-N_{i}}, N_{r}, N_{0}\right]^{T}
$$

This parameter vector was estimated using the maximum likelihood method for all the runs 221-225. The parameter values obtained were used to generate Tables 2 and 3.

\subsection{Discussion of results}

The parameter values obtained during the analysis of the captive and the freerunning model tests are presented in Tables 2 and 3.

Because the model structure in eqn. (6) does not contain information of the inertia coefficients, Table 2 contains parameters of equations on the form:

$$
\left.\begin{array}{l}
\ddot{v}^{\prime}=Y^{\prime}{ }_{v} v^{\prime}+Y_{r}^{\prime} r^{\prime}+Y^{\prime}{ }_{\delta} \delta^{\prime}+Y^{\prime}{ }_{0} \\
\ddot{r}^{\prime}=N_{v}^{\prime} v^{\prime}+N^{\prime}{ }_{v|p|} \cdot v^{\prime}\left|v^{\prime}\right|+N_{r}^{\prime} r^{\prime}+N_{\delta}^{\prime} \delta^{\prime}+N_{0}^{\prime}
\end{array}\right\}
$$

where all quantities are in non-dimensional form, according to the 'prime' system. This system is described in Abkowitz (1964). 

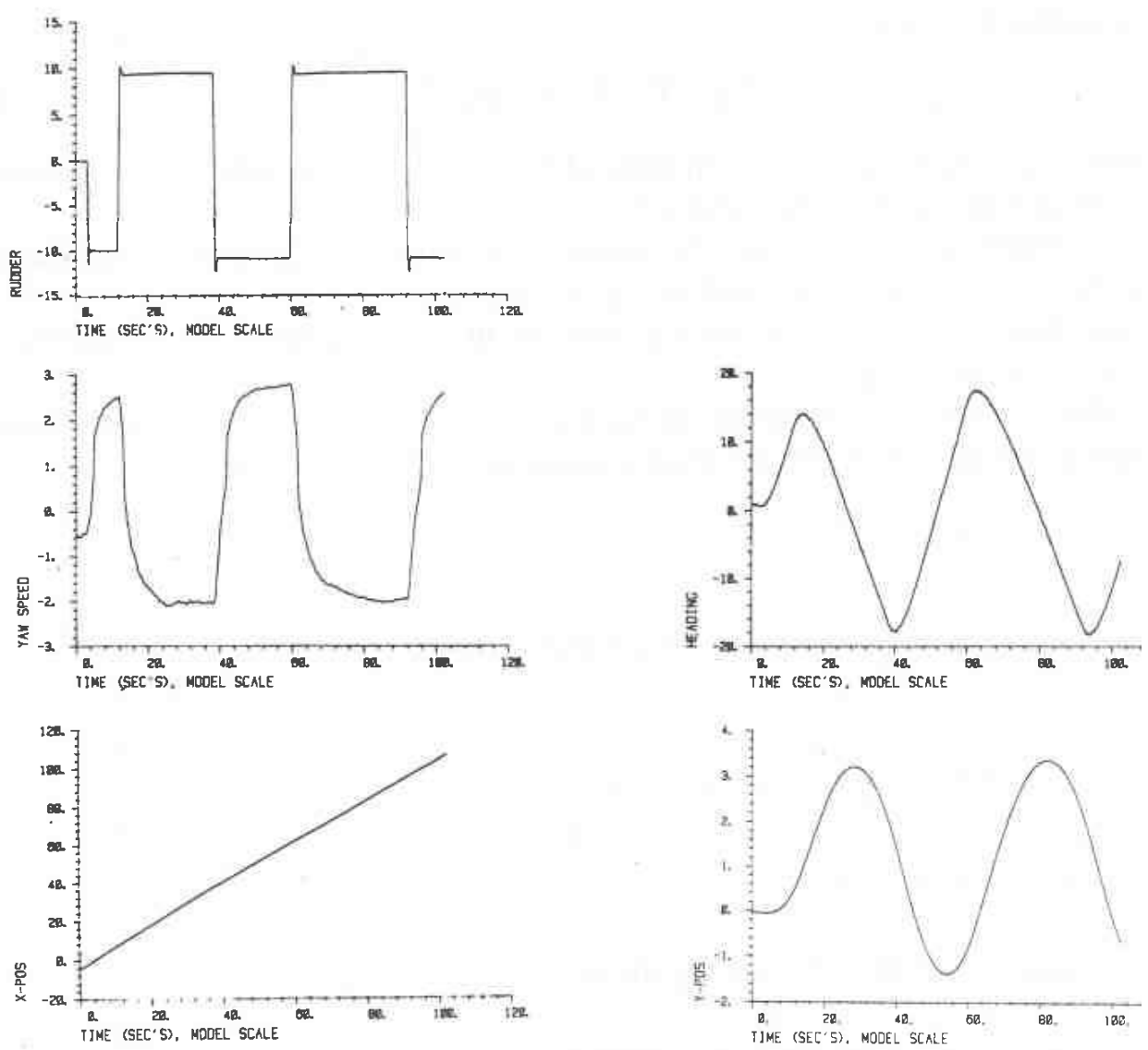

Figure 6. Measurements for run number 221. Rudder: rudder angle [degrees]; speed: yaw speed [degrees/s]; Heading: heading angle [degrees]; $X$-pos, $Y$ global coordinates [M].

Because the inertia coefficients could not be determined using the model stru in eqn. (6), parameters of eqn. (12) are given instead of in a model structure eqn. (1). Table 2 contains the parameters obtained using run number 221 anc model in eqn. (6). This set of parameters is called: 221 eq. 6 . Then it contain parameters obtained with the model in eqn. (9) for all runs. These parameter are called: 221-225. The last set of parameters in Table 2, denoted PMM, is $t$ from Jacobsen and Terslov (1979). This set contains the parameters from pl motion testing of the same ship. It should, however, be noted that the PMM were performed at another water line than the tests described in this paper. fortunately this means that the results are not directly comparable, however, reasonable to expect them to be within the same region.

The parameters for the first 3 runs correspond quite well, the last 2 not that The excitation was \pm 10 degrees for runs no. 221-223, -6 to +4 degrees for 224 , and -7 to +3 degrees for run 225 . The greater excitations for runs 221 increases the reliability of the parameter estimates. Using the model in eqn. (6) parameters $Y_{v}, N_{r}$ take up the effect both from sway- and yaw-speed, giving diff parameter values. As we shall see this does not mean that the response of the mor wrong. Table 3 presents the inertia coefficients obtained during estimation 0 model in eqn. (9). 


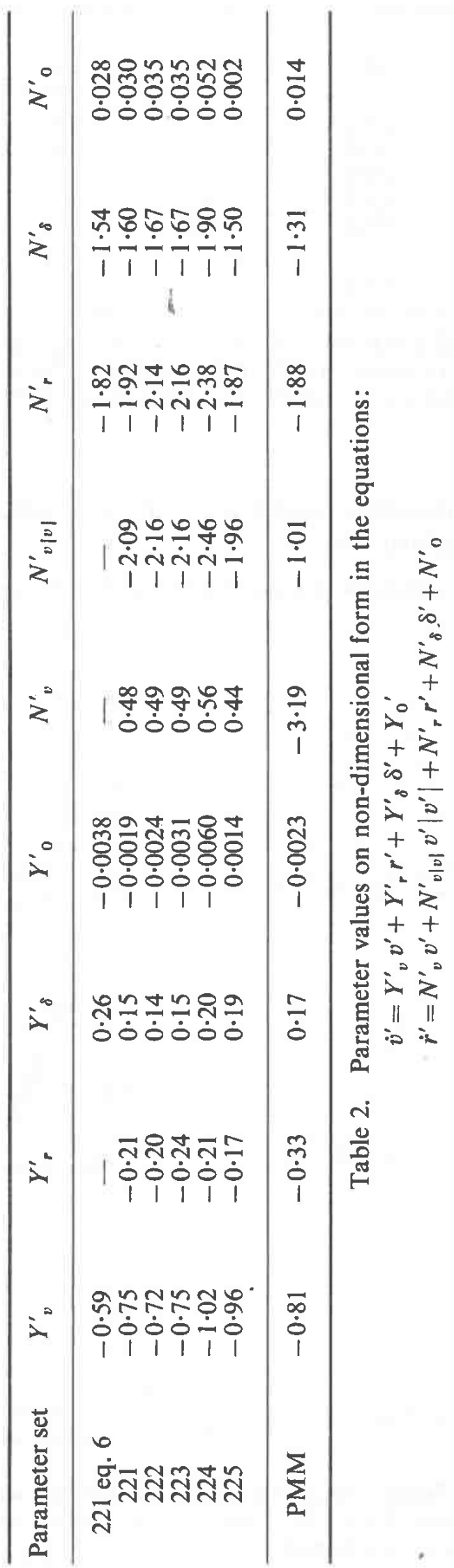




\begin{tabular}{ccccc}
\hline $\begin{array}{c}\text { Parameter } \\
\text { set }\end{array}$ & $m^{\prime}$ & $Y^{\prime}\{$ & $l^{\prime}{ }_{0}$ & $N^{\prime}{ }^{\prime}$ \\
\hline 221 & 1252 & -1326 & 50 & -61 \\
222 & 1252 & -1435 & 50 & -57 \\
223 & 1252 & -1326 & 50 & -57 \\
224 & 1252 & -656 & 50 & -44 \\
225 & 1252 & -735 & 50 & -69 \\
\hline PMM & 1358 & -1129 & 78 & -65
\end{tabular}

Table 3. Inertia coefficients on non-dimensional form. The inertia coefficients : calculated from the estimated coefficients using eq. (9) for the 5 runs 221-2. $m^{\prime}$ and $I^{\prime}{ }_{0}$ are weight and moment of inertia in air.

\section{Simulations}

To verify the mathematical models obtained, simulations have been perform The manoeuvres simulated were:

The same zig-zag manoeuvres used during the model tests.

Turning circles.
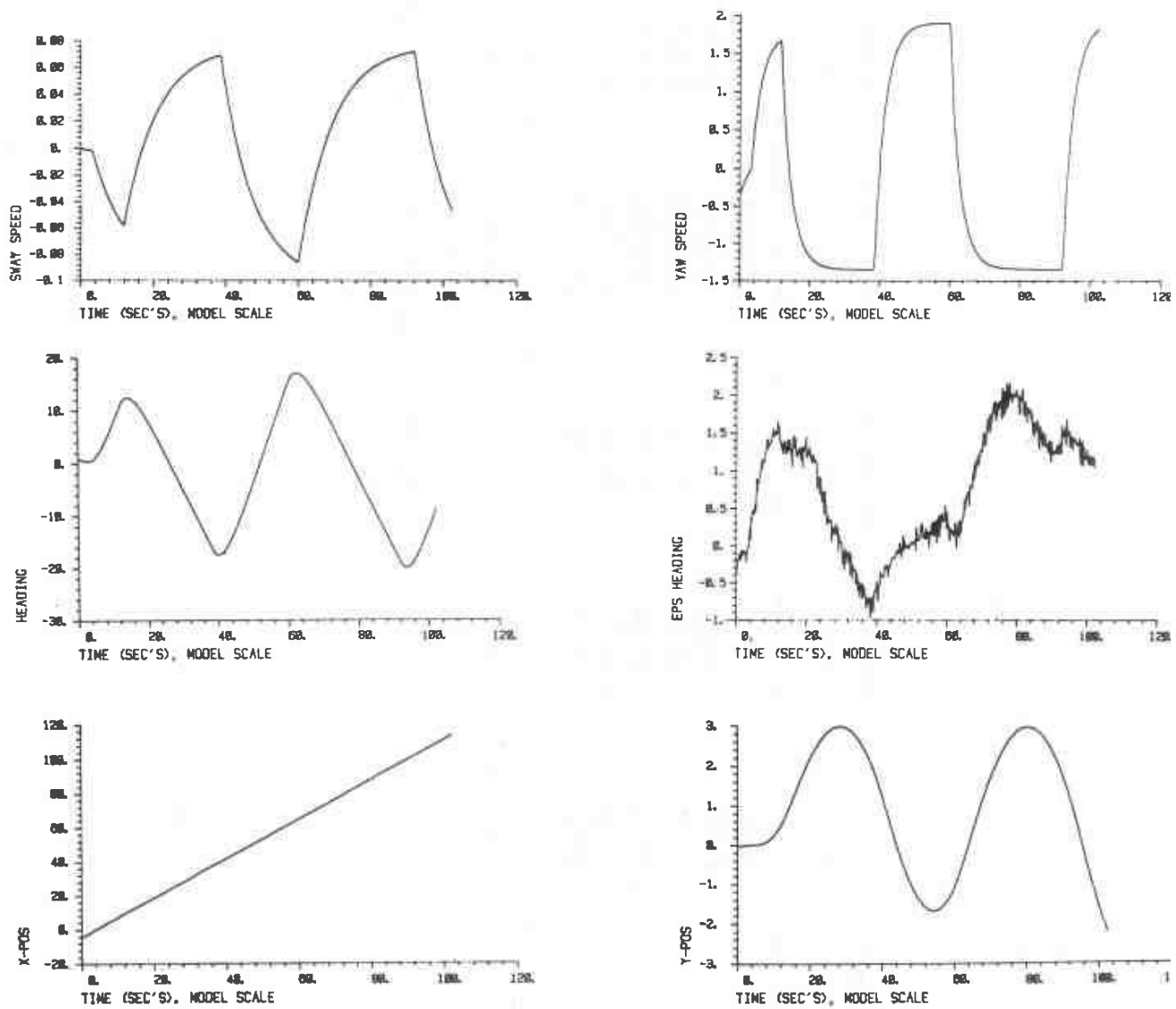

Figure 7. Simulation results. Simulation of run number 221 , with the model in eqn. 1 and parameter set: 221 eq. 6 (Table 1). EPS heading: measured heading angl simulated heading angle [degrees]. 
188

T. Flobakk
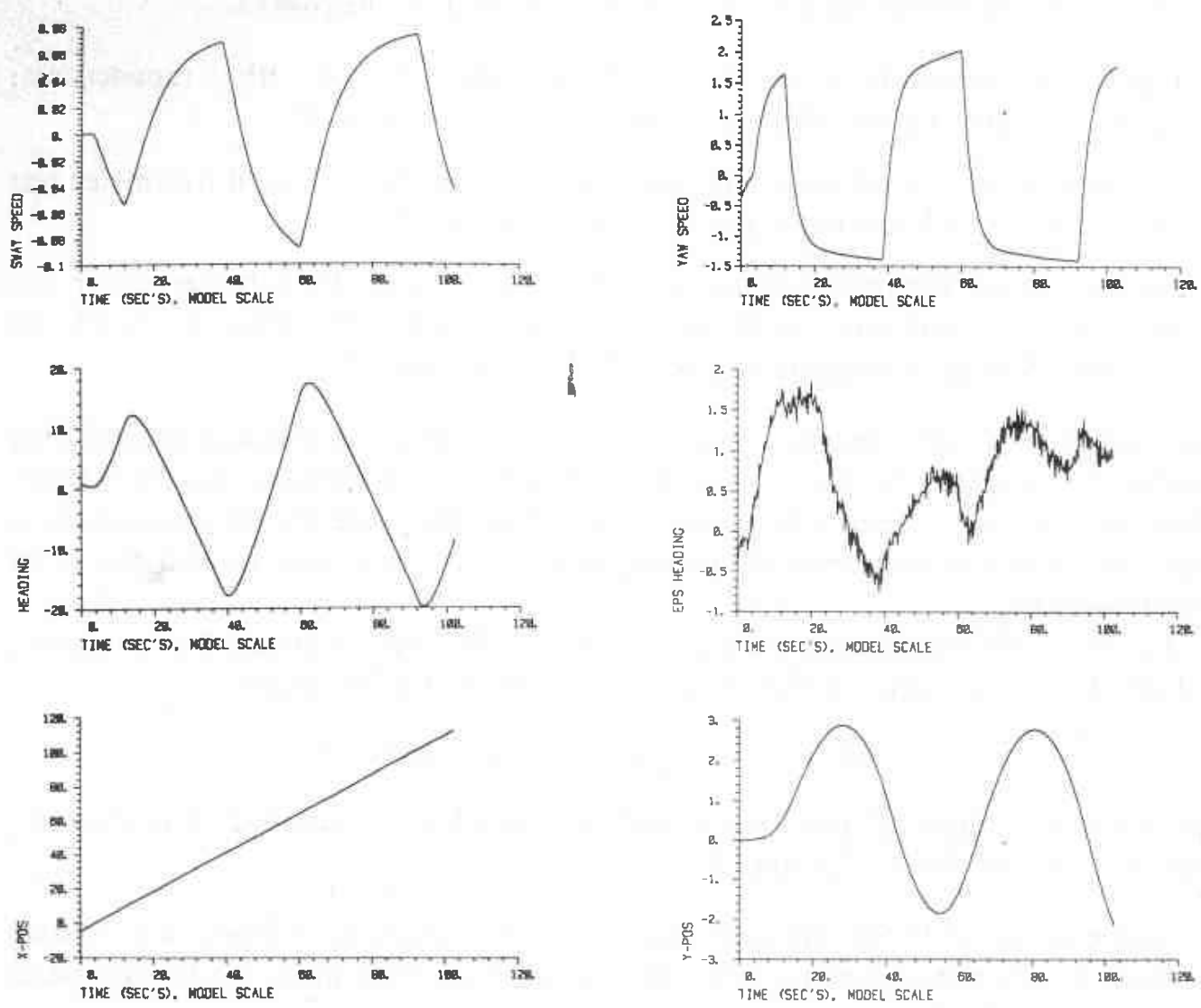

Figure 8. Simulation results. Simulation of run number 221, with the model in eqn. (9), and parameter set: 221 (Table 1).
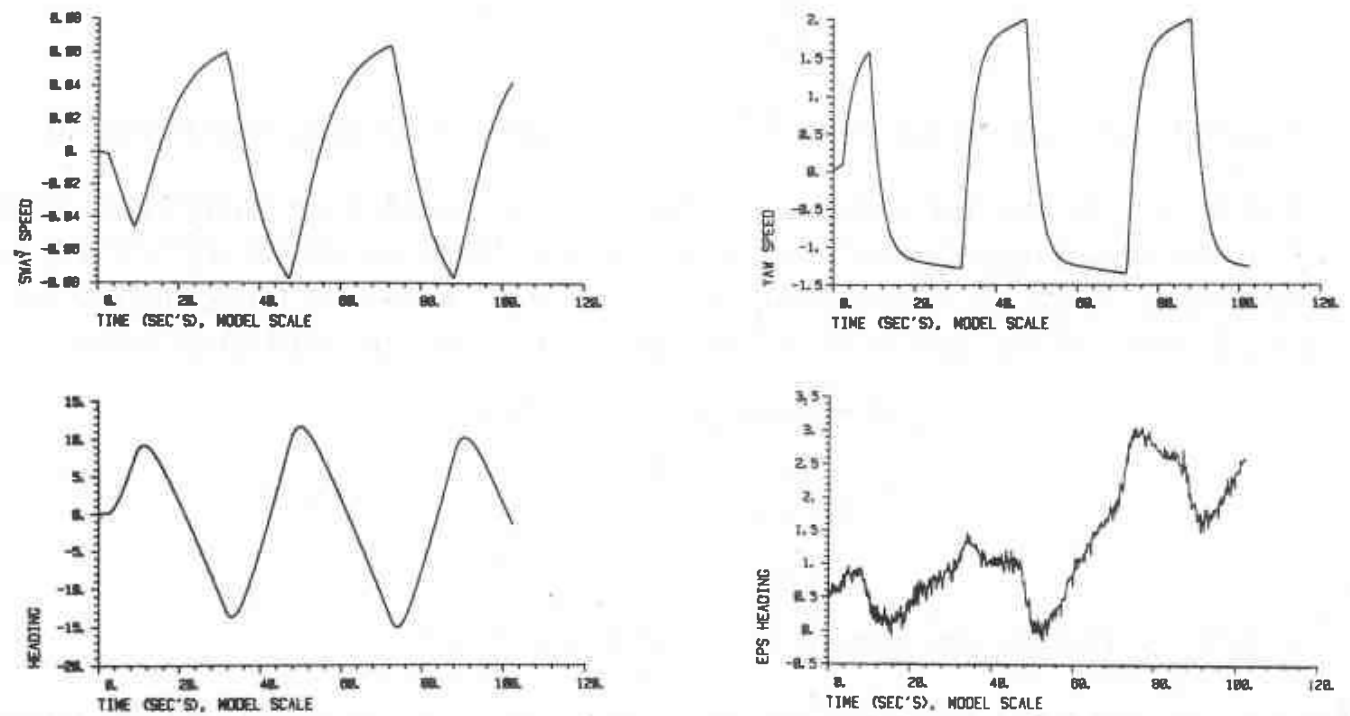

Figure 9. Simulation results. Simulation of run number 222, with the parameters for run number 221. 
Three simulations of zig-zag manoeuvres are presented in this paper:

Figure 7 shows simulation results with the model in eqn. (6), with parameters set: 221 eq. 6 (Table 1), and the rudder signal for run number 221.

Figure 8 shows simulation results with the model in eqn. (9), with parameter set: 221 (Table 1), and the rudder signal for run number 221.

Figure 9 shows simulation results with the model in eqn. (9), with parameter set: 221 (Table 1), and the rudder signal for run number 222. (Simulation of run number 222 with parameters estimated for run number 221.)

The various states are presented, and also the deviation of the heading angle during simulation compared to the measurement, denoted EPS Heading on the figures. Observing Figs. 6, 7, 8 and 9 both mathematical models represent the measurements quite well. Also the parameters estimated from one run, represent another run in an acceptable way.

Turning circle simulations were performed for the 6 sets of parameters presented in Table 1. For each set, simulation was done with the rudder angles

$$
\delta=0, \pm 1, \pm 2, \pm 5, \pm 10, \pm 15 \text { [degrees] }
$$

The stationary values of sway speed and yaw speed were recorded, and these are presented on Figs. 10, 11, 12 and 13.

Figure 10 presents the stationary values of sway speed as a function of rudder angle for the parameter sets $221-225$ in Table 1. Arrows mark the various parameter sets in the following way:

$\begin{array}{cc}\text { Run no. } & \text { Mark } \\ 221 & \leftarrow \\ 222 & \swarrow \\ 223 & \downarrow \\ 224 & \searrow \\ 225 & \rightarrow\end{array}$

Figure 11 presents the stationary values of yaw speed in the same way as Fig. 10.

Figure 12 presents the stationary values of sway speed from simulations with 3 mathematical models and 3 sets of parameters. These are set 221 and 221 eq. 6 , and the complete non-linear model obtained from PMM-tests, found in Jacobsen and Terslov (1979). To identify the parameter sets these are marked as follows:

$\begin{array}{cc}\text { Parameter set } & \text { Mark } \\ 221 & \leftarrow \\ 221 \text { eq. } 6 & \downarrow \\ \text { PMM } & \rightarrow\end{array}$

Figure 13 presents yaw speed in the same way as Fig. 12.

The various parameter sets identified gave similar responses simulating turning circle manoeuvres. These responses were different from the PMM results, but as earlier mentioned, testing was done at different waterlines. 


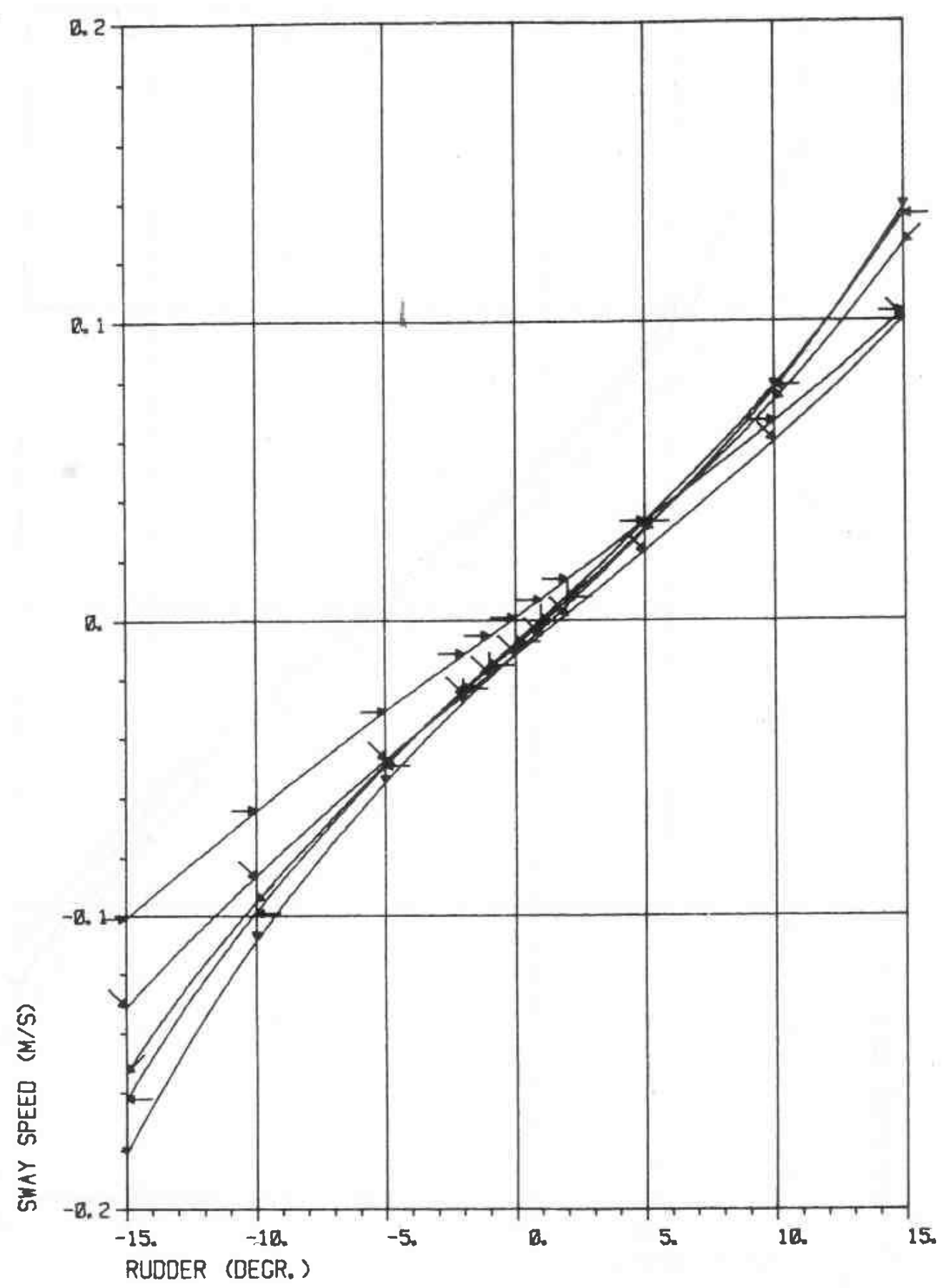

Figure 10. Simulation of turning circles. Stationary values of sway speed for different rudder angles. The simulations are done with the model in eqn. (9) and the parameter sets $221-225$ in Table 1 . To identify the parameter sets, the following marks are used:

$\begin{array}{cc}\text { Parameter set } & \text { Mark } \\ 221 & \leftarrow \\ 222 & \\ 223 & \downarrow \\ 224 & \vdots \\ 225 & \rightarrow\end{array}$




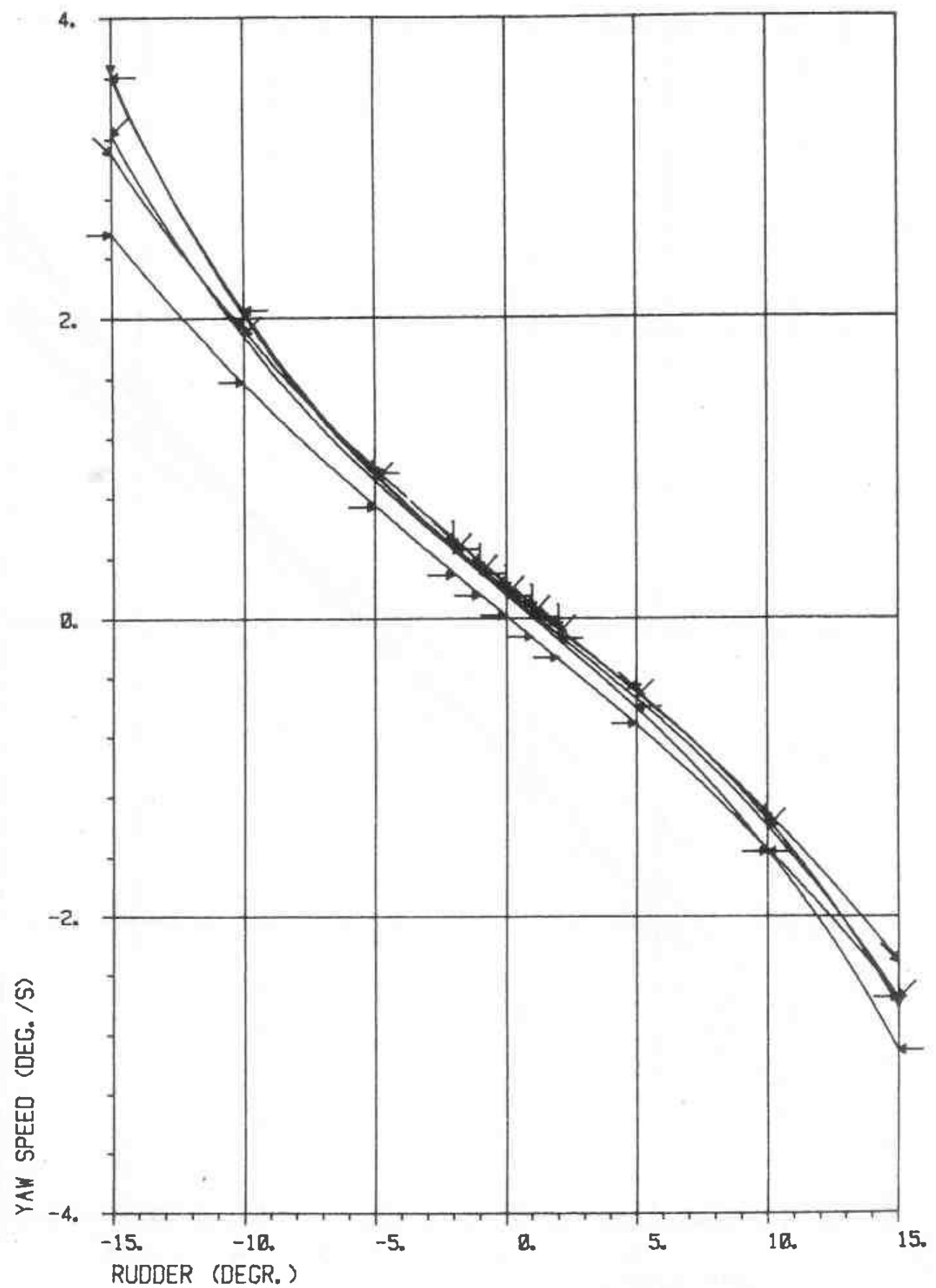

Figure 11. Simulation of turning circles. Stationary values of yaw speed for different rudder angles. The simulations are done with the model in eqn. (9) and the parameter sets 221-225 in Table 1. To identify the parameter sets, the following marks are used:

Parameter set
221
222
223
224
225

Mark

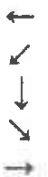




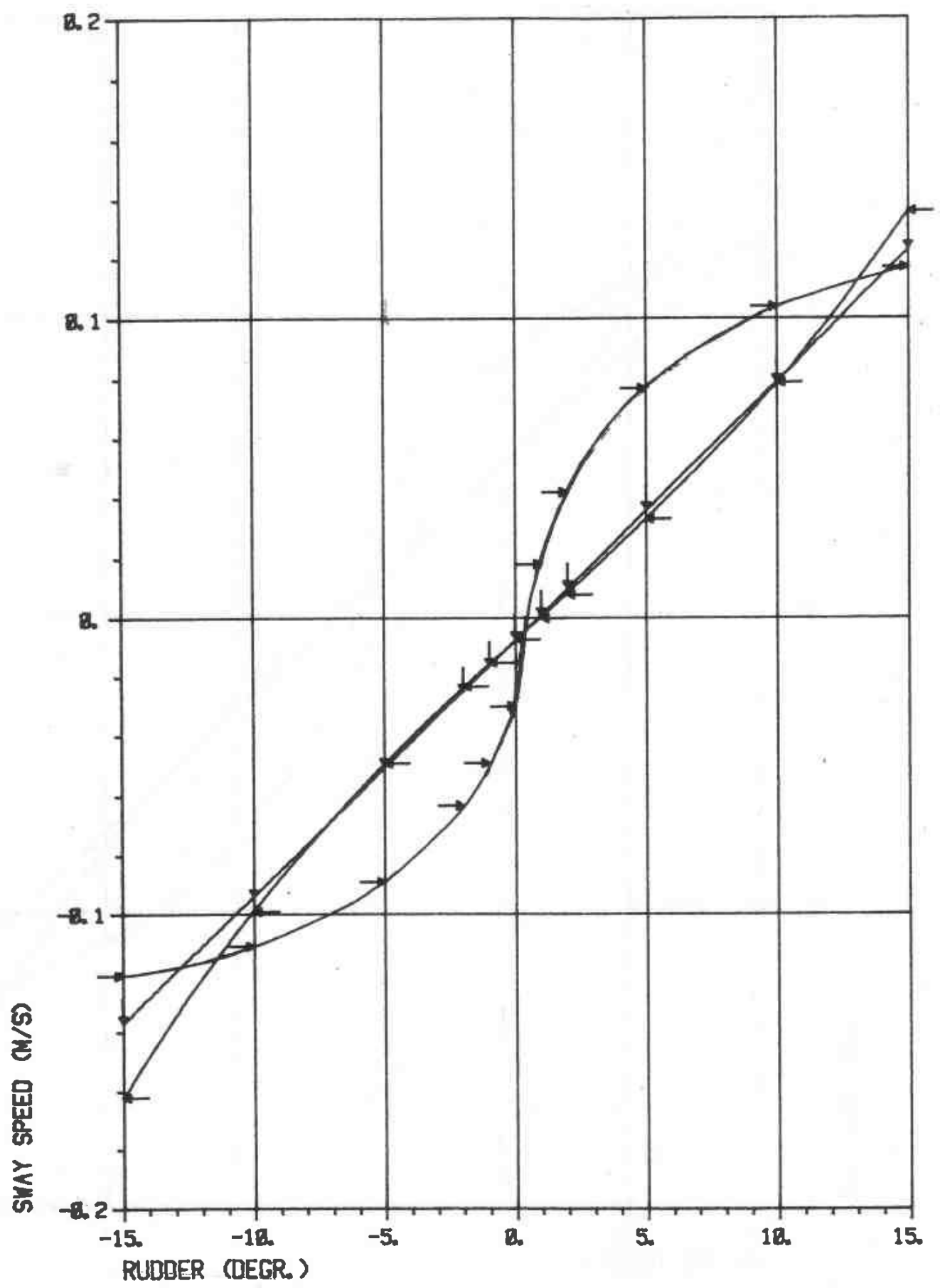

Figure 12. Simulation of turning circles. Stationary values of sway speed for different rudder angles. The simulations are done with 3 mathematical models and parameter sets: Model in eqn. (9), and parameter set: 221 in Table 1; Model in eqn. (6), and parameter set: 221 eq. 6 in table 1; Complete non-linear model obtained from PMM tests (Jacobsen and Terslov 1979).

To identify the parameter sets, the following marks are used:

$\begin{array}{cc}\text { Parameter set } & \text { Mark } \\ 221 \text { - } & \leftarrow \\ 221 \text { eq } 6 & \downarrow \\ \text { PMM } & \rightarrow\end{array}$




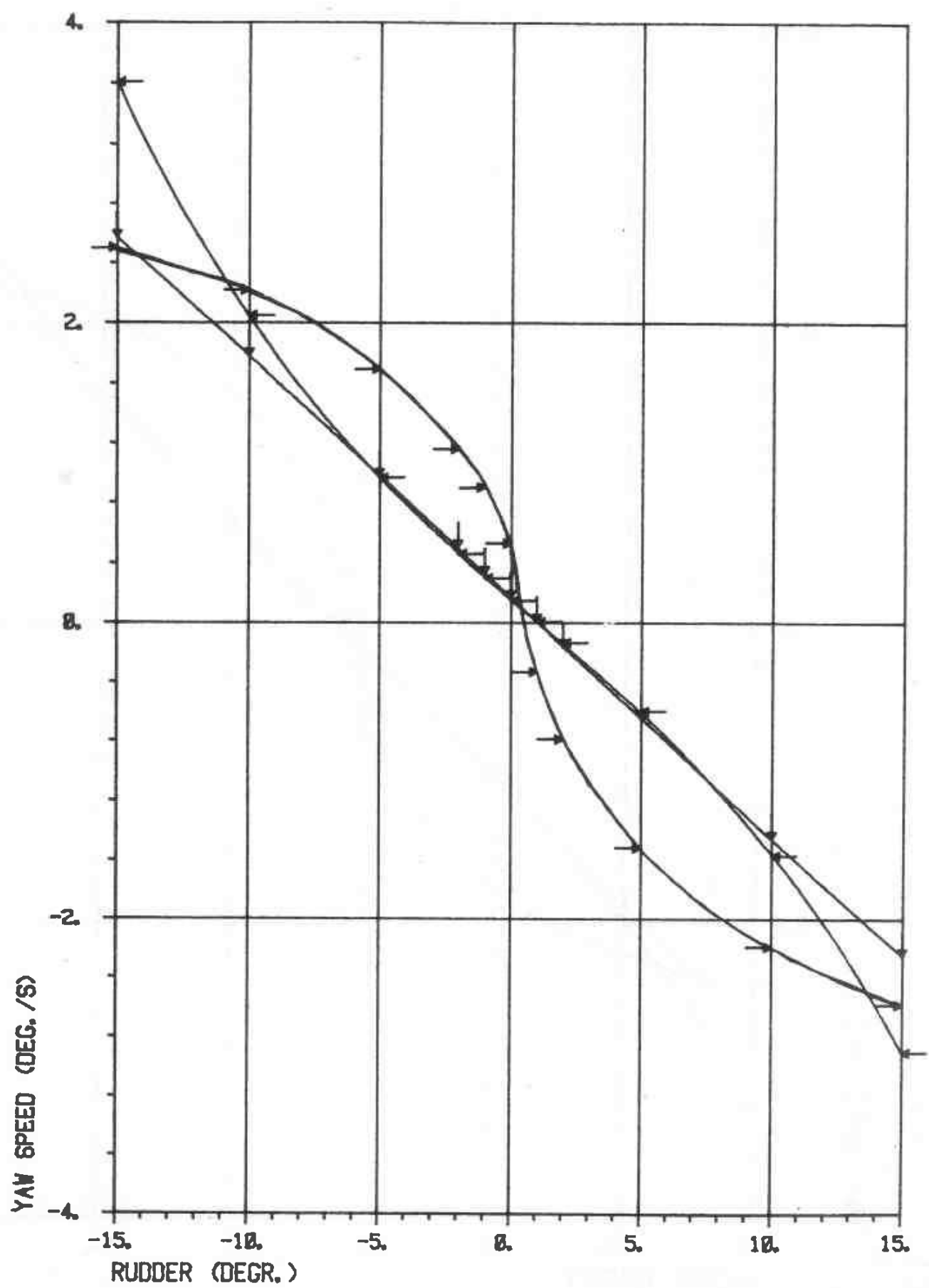

Figure 13. Simulation of turning circles. Stationary values of yaw speed for different rudder angles. The simulations are done with 3 mathematical models and parameter sets: Model in eqn. (9), and parameter set: 221 in Table 1; Model in eqn. (6), and parameter set: 221 eq. 6 in Table 1; Complete non-linear model obtained from PMM tests (Jacobsen and Terslov 1979).

To identify the parameter sets, the following marks are used:

$\begin{array}{cc}\text { Parameter set } & \text { Mark } \\ 221 \text { - } & \leftarrow \\ 221 \text { eq } 6 & \downarrow \\ \text { PMM } & \rightarrow\end{array}$




\section{Conclusion}

Combined captive and free-running model testing gives the parameters of the manoeuvring equations for ships using small rudder deflections. With the new ocean laboratory at NHL, the method will be adapted to identify non-linear models covering larger rudder deflections. Free-running tests alone give a mathematical model with correct response, but the inertia parameters are not detected. To identify complete mathematical models of ships, NHL is using a combination of theoretical calculations of added mass coefficients, oblique towing tests and free-running tests.

\section{REFERENCES}

Aвкоwiтz, M. A. (1964). Lectures on ship hydrodynamics-Steering and manoeuvrability. Report no. Hy-5. Hydrodynamics Department, Hydro- and Aerodynamics Laboratory, Lyngby, Denmark.

Åstrøm, K. J., and EyкHOff, P. (1971). System identification-A survey. Automatica, 7, 123.

Åstrøm, K. J., Källstrøm, C. G., Norrbin, N. H., and Bystrøm, L. (1975). The identification of linear ship steering dynamics using maximum likelihood parameter estimation. Publ. no. 75, Swedish State Shipbuilding Experimental Tank, Gothenburg.

Åstrøм, K. J., and KällstrøM, C. G. (1976). Identification of ship steering dynamics. Automatica, 12, 9.

BerG, T. E., and FlobaKK, T. (1979). Determination of coefficients in the manoeuvring equations of ship. Norwegian Maritime Research, 7.

BlaNKE, M. (1978). On identification of nonlinear speed equation from full scale trials. Proc. 5th Ship Control Systems Symp., Vol. 6, Annapolis, Maryland, USA

Chislett, M. S., and STRøM-TejSen, J. (1965). Planer motion mechanism tests and full-scale steering and manoeuvring predictions for a Mariner class vessel. Report no. Hy-6. Hydrodynamics Department, Hydro- and Aerodynamics Laboratory, Lyngby, Denmark.

EDA, H., and CRAINE, C. L. (1967). Non-linear prediction of steering performance of 60 models. Report no. 1980, 1967. Davidson Laboratory, New Jersey.

EDA, H. (1978). A digital simulation study of steering control with effects of roll motion. 5th Ship Control Systems Symposium, Annapolis 1978.

FlobaKK, T. (1982). Parameter estimation techniques applied to ship manoeuvring tests in model scale. Thesis. The Norwegian Institute of Technology. Division of Engineering Cybernetics, Trondheim, Norway.

HALlingSTAD, O. (1976). Maksimum likelihood identifikasjon av parametre $i$ ulineare tilstandsrommodeller (in Norwegiàn). SINTEF report STF 48 A76071, Trondheim, Norway.

JACOBSEN, B. K., and Terslov, O. (1979). Steering and manoeuvring tests with a model of a $127 \mathrm{~m}$ gas tanker. SL report 79067. Hydrodynamics Department, Hydro- and Aerodynamics Laboratory, Lyngby, Denmark.

KäLlstrøm, C. G. (1979). Identification and adaptive control applied to ship steering. Thesis. Department of automatic control. Lund Institute of Technology, Lund, Sweden.

Newman, J. N. (1977). Marine hydrodynamics (MIT Press: Cambridge, Massachusetts).

NORRBIN, N. H. (1970). Theory and observations on the use of a mathematical model for ship manoeuvring in deep and confined waters. 8th Symposium on Naval Hydrodynamics, Pasadena, California.

Rossing, N. KR. (1981). Akustisk posisjoineringssystem (in Norwegian). SINTEF report STF44 F91133, Trondheim, Norway.

SfeLID, S. (1975). Systemidentifikasjon ved maksimum likelihood-metoder (in Norwegian). SINTEF report: STF48 A75048, Trondheim, Norway. 